\title{
Reconstitution of huPBL-NSG Mice with Donor-Matched Dendritic Cells Enables Antigen-Specific T-cell Activation
}

\author{
Airi Harui • Sylvia M. Kiertscher • Michael D. Roth
}

Received: 1 April 2010 /Accepted: 18 May 2010 /Published online: 8 June 2010

(C) The Author(s) 2010. This article is published with open access at Springerlink.com

\begin{abstract}
Humanized mouse models provide a unique opportunity to study human immune cells in vivo, but traditional models have been limited to the evaluation of non-specific T-cell interactions due to the absence of antigen-presenting cells. In this study, immunodeficient NOD/SCID/IL2r- $\gamma^{\text {null }}$ (NSG) mice were engrafted with human peripheral blood lymphocytes alone or in combination with donor-matched monocyte-derived dendritic cells (DC) to determine whether antigen-specific T-cell activation could be reconstituted. Over a period of 3 weeks, transferred peripheral blood lymphocytes reconstituted the spleen and peripheral blood of recipient mice with predominantly human CD45-positive lymphocytes. Animals exhibited a relatively normal CD4/CD8 ratio (average $1.63: 1$ ) as well as reconstitution of $\mathrm{CD} 3 / \mathrm{CD} 56$ (averaging $17.8 \%$ ) and CD20 subsets (averaging 4.0\%). Animals
\end{abstract}

This study was presented at the 15 th Scientific Conference of the Society on NeuroImmune Pharmacology, Wuhan, China, 2009.

This study was supported by funding from the NIH/National Institute on Drug Abuse (RO1 DA03018), the Department of Defense Prostate Cancer Research Program, Stewart and Lynda Resnick, the Prostate Cancer Foundation, the UCLA Department of Urology, and Core support from the Jonsson Comprehensive Cancer Center.

A. Harui · S. M. Kiertscher $\cdot$ M. D. Roth

Division of Pulmonary \& Critical Care Medicine,

David Geffen School of Medicine at UCLA,

Los Angeles, CA 90095-1690, USA

M. D. Roth $(\bowtie)$

Division of Pulmonary \& Critical Care Medicine,

Department of Medicine, David Geffen School of Medicine at

UCLA,

Los Angeles, CA 90095-1690, USA

e-mail: mroth@mednet.ucla.edu reconstituted with donor-matched CD11c+ DC also demonstrated a $\mathrm{CD} 11 \mathrm{c}+$ population within their spleen, representing $0.27 \%$ to $0.43 \%$ of the recovered human cells with concurrent expression of HLA-DR, CD40, and CD86. When immunized with adenovirus, either as free replication-incompetent vector (AdV) or as vectortransduced DC (DC/AdV), there was activation and expansion of AdV-specific T-cells, an increase in Th1 cytokines in serum, and skewing of T-cells toward an effector/memory phenotype. T-cells recovered from animals challenged with $\mathrm{AdV}$ in vivo proliferated and secreted a Th1-profile of cytokines in response to DC/AdV challenge in vitro. Our results suggest that engrafting NSG mice with a combination of lymphocytes and donor-matched DC can reconstitute antigen responsiveness and allow the in vivo assessment of human immune response to viruses, vaccines, and other immune challenges.

Keywords dendritic cell · adenovirus · vaccination · xenotransplant $\cdot$ cytokine $\cdot$ humanized mouse

\section{Introduction}

While inbred strains of conventional and genetically modified mice have provided an unparalleled opportunity to examine the process of antigen presentation and the characteristics of adaptive T-cell immunity, translating these findings into new approaches for human immunotherapy has proven problematic (Mestas and Hughes 2004; Ju et al. 2010). Responses to cytokine therapy, vaccines, gene therapy, adjuvants, and adoptive cell transfers that are reproducible and robust in the mouse are often rare when translated into human clinical trials. This is not surprising given that human dendritic cells (DC) are distinctively 
different from those of the mouse, as are the antigens of interest, the major histocompatibility complex (MHC) repertoire, toll-like receptor expression, cytokine responses, and many other features. Genetic diversity is also a major factor, as are the susceptibility and prior exposure to many human pathogens and immune challenges (Mestas and Hughes 2004; Zaiss et al. 2009). Even transgenic animals that express a human MHC haplotype or spontaneously develop organ-specific tumors have not proven to be predictive of integrated human immune responses (Pascolo et al. 1997).

One approach for bridging the gap between mouse models and human clinical trials has been to develop humanized mice-immunodeficient strains which permit the engraftment and expansion of human immune cells in vivo (Shultz et al. 2007; Pearson et al. 2008). A variety of different humanized mouse models have been developed and fall into two distinct categories: (1) those with a slow but more extensive immune reconstitution resulting from stem cell transfer (Lapidot et al. 1992; Greiner et al. 1998; Ito et al. 2002) or (2) those with rapid reconstitution based on the transfer of fully differentiated human peripheral blood cells (Mosier et al. 1988; Hesselton et al. 1995; May et al. 2005). We have focused on the latter, not only for the speed of the reconstitution but for the prospect that the reconstituted immune system would reflect the immunologic memory of the human donor. For these studies, immunodeficient NOD/SCID-IL2r $\gamma^{\text {null }}$ (NSG) mice, which lack the $\gamma$-chain of the IL-2 receptor in addition to the NOD/SCID background, were chosen (Ishikawa et al. 2005; Shultz et al. 2005). NSG mice lack high affinity receptors for interleukin (IL)-2, IL-4, IL-7, IL-9, IL-15, and IL-21. As a result of this ineffective cytokine signaling, these mice completely lack functional T-cells, B-cells, NK cells, macrophages, and DC and allow nearly complete immune reconstitution by engrafted human cells (Ishikawa et al. 2005; Shultz et al. 2005). We hypothesized that humanized NSG animals, when reconstituted with both peripheral blood lymphocytes (PBL) and with donormatched antigen presenting DC, would allow us to examine the process of human antigen presentation and the activation of antigen-specific T-cells as it occurs in vivo, in the context of the immunologic repertoire of the human donor. In these studies, we have used a replicationincompetent serotype-5 adenoviral vector ( $\mathrm{AdV}$ ) as the antigen. Human exposure to adenovirus and the development of anti-viral memory T-cell responses occurs with a high frequency in the adult population (Olive et al. 2001; Roth et al. 2002). As predicted, only human peripheral blood lymphocyte (huPBL)-NSG mice that were reconstituted with both PBL and DC demonstrated AdV-specific Tcell proliferation and cytokine production. Furthermore, the characteristics of the AdV-specific response recapitulated those of the originally implanted PBL. This model should provide a rapid and highly flexible system for studying the activation of human antigen-specific immunity and provide a novel platform for vaccine development and testing, as well as for the study of interactions between antigenspecific immunity and a variety of host challenges such as viral exposures (including HIV), tumors, and systemic drug effects.

\section{Materials and methods}

Animals

Six- to 8-week-old NSG mice were obtained from The Jackson Laboratories (Bar Harbor, ME, USA) and housed at the UCLA Mouse/Human Chimera Core Facility under laminar flow conditions with all food, water (acidified), caging, and bedding autoclaved before use. No antibiotics were administered. All procedures were approved by the UCLA Animal Research Committee.

\section{Culture media and reagents}

Cells were cultured in complete medium composed of RPMI 1640 (Mediatech, Manassas, VA, USA) supplemented with $10 \%$ heat-inactivated human $\mathrm{AB}$ serum (Omega Scientific, Tarzana, CA, USA), penicillinstreptomycin-fungizone (Invitrogen, Carlsbad, CA, USA), and $10 \mathrm{mM}$ HEPES (Sigma Chemical, St. Louis, MO, USA). Monoclonal antibody (mAb) combinations used for the enrichment of T-and B-cells (anti-CD14, antiCD16, and anti-CD25) and DC (anti-CD3, anti-CD16, and anti-CD19) by negative depletion were obtained from BD Biosciences (San Jose, CA, USA). Anti-mouse Ig-conjugated immunomagnetic beads were from Invitrogen (M450 Dynabeads; Carlsbad, CA, USA). Fluorochrome-conjugated mAbs against CD3, CD4, CD8, CD11c, CD86, and HLA-DR were obtained from BD Biosciences; anti-CD127 and anti-GITR from eBiosciences (San Diego, CA, USA); and anti-CD25 from BioLegend (San Diego, CA, USA). T-cell proliferation in vitro was evaluated using carboxyfluorescein diacetate, succinimidyl ester (CFSE; Vybrant CFDA SE Cell Tracer Kit, InvitrogenMolecular Probes, Eugene, OR, USA).

\section{Adenoviral vectors}

A replication-deficient adenoviral vector (AdV) expressing green fluorescent protein (GFP) and modified to express an additional Arg-Gly-Asp peptide sequence in the HI loop region of their fiber knob was obtained from Mizuguchi et 
al. (2001) (AdV-GFP). A replication-deficient adenoviral vector with a matching CMV promoter but without an inserted transgene sequence (AdV-RR5) was also used as previously described (Roth et al. 2002). Adenoviral vectors were propagated in 293 cells, purified by CsCI density centrifugation, dialyzed, and stored in $-80^{\circ} \mathrm{C}$. Viral particle titers were determined spectrophotometrically (Mittereder et al. 1996).

Generation and transduction of donor-matched DC

Peripheral blood mononuclear cells (PBMC) were obtained from healthy normal donors by density gradient centrifugation and DC prepared by culturing the adherent fraction with $800 \mathrm{IU} / \mathrm{ml}$ granulocyte-macrophage colony stimulating factor (GM-CSF; Bayer Healthcare, Seattle, WA, USA) and $63 \mathrm{ng} / \mathrm{ml} \mathrm{IL-4} \mathrm{(R \& D} \mathrm{Systems,} \mathrm{Minneapolis,} \mathrm{MN,}$ USA) for 5 days at $37^{\circ} \mathrm{C}$ as previously described (Kiertscher and Roth 1996). DC were recovered on day 5 and purified by negative depletion using a mAb cocktail and anti-mouse Ig-conjugated immunomagnetic beads. DC were either used fresh or aliquoted and cryopreserved in medium containing $20 \% \mathrm{AB}$ serum and $10 \%$ DMSO. Fresh or cryopreserved DC that had been thawed and cultured for 8-24 h in complete media containing GM-CSF and IL-4 were transduced with $\mathrm{AdV}$ as described previously (Harui et al. 2006a, b). Briefly, 5.0×105 DC suspended in $100 \mu \mathrm{l}$ of complete medium were exposed to a suspension of $\mathrm{AdV}$ in phosphate-buffered saline (PBS) containing calcium and magnesium at a multiplicity of infection (MOI) of 4,000 viral particles/cell. Cultures were incubated for $2 \mathrm{~h}$ at $37^{\circ} \mathrm{C}$ in a humidified incubator and an additional $800 \mu \mathrm{l}$ of complete media containing GM-CSF and IL-4 was then added. Human MegaCD40L (200 ng/ml, Alexis Biochemicals, Plymouth Meeting, PA, USA) and interferon gamma (IFN- $\gamma ; 100$ U/ml, PeproTech, Rocky Hills, NJ, USA) were added into culture media $24 \mathrm{~h}$ later to stimulate a mature DC phenotype, and cells were incubated for an additional $24 \mathrm{~h}$ and harvested.

\section{Reconstitution and vaccination of NSG mice}

A combination of $\mathrm{T}$-and $\mathrm{B}$-cells were purified from PBMC by negative depletion using a mAb cocktail and anti-mouse Ig-conjugated immunomagnetic beads. Cells were resuspended in PBS and administered to recipient NSG mice $\left(10^{7}\right.$ cells/mouse) by intraperitoneal (i.p.) injection on day 0 of the reconstitution. To adapt huPBL-NSG mice as a platform for assessing human antigen-specific T-cell activation, animals were also administered an i.p. injection on day 0 of $0.5 \times 10^{6} \mathrm{DC}$ that had been generated from the same donor (donormatched) (Fig. 1). Monocyte-derived DCs were adminis- tered as either control DC (which had not been exposed to AdV in vitro) along with $1 \times 10^{9}$ or $1 \times 10^{10}$ free AdV-GFP particles, or as DC that had previously been transduced with the AdV-GFP vector in vitro (DC/AdV-GFP). For some experiments, animals were administered a second injection of donor-matched DC that had been prepared from the same donor (either DC/AdV-GFP or control DC in combination with free viral particles) 7 days later using the same methods.

Cell recovery and analysis of human cells by flow cytometry

Single-cell suspensions were prepared from the spleen by mechanical disaggregation and from the peritoneal cavity by lavage followed by depletion of red blood cells by hypotonic shock. Cells were stained with fluorochrome-conjugated $\mathrm{mAbs}$ and analyzed by flow cytometry (FacsCalibur, Becton-Dickinson, San Jose, CA, USA) in combination with FCS Express analysis software (DeNovo Software, Los Angeles, CA, USA). Human cells were defined by expression of human CD45 and subsets identified by specific marker expression for T-cell subsets $(\mathrm{CD} 3+/ \mathrm{CD} 4+$ or $\mathrm{CD} 3+/ \mathrm{CD} 8+$ alone or in combination with GITR, CD127, and/or CD25), B-cells (CD20), NK cells (CD3-/CD56+), NK-T-like cells (CD3+/CD56+), or DC (CD11c, CD40, CD86, and/or HLA$\mathrm{DR})$.

\section{Detection of antigen-specific T-cell responses}

Recovered spleen cells were challenged in vitro with donor-matched control DC or with DC/AdV-RR5 to detect responses to adenoviral capsid antigens. Antigenspecific responses were assessed by intracellular cytokine stimulation (ICCS), proliferation, or cytokine secretion assays. For ICCS, $2 \times 10^{6}$ spleen cells were stimulated with either control DC or DC/AdV-RR5 at a ratio of 1:10 (DC/spleen cells ratio) in complete media in the presence of $3 \mu \mathrm{g} / \mathrm{ml}$ anti-CD28 Ab (BD Biosciences) and $1 \mathrm{ng} / \mathrm{ml}$ IL-12 (PeproTech) for $5 \mathrm{~h}$ at $37^{\circ} \mathrm{C}$. Brefeldin A (SigmaAldrich, St. Louis, MO, USA) was added at $10 \mu \mathrm{g} / \mathrm{ml}$ after the first hour. At the end of the culture period, cells were fixed and cryopreserved. Intracellular staining for IFN- $\gamma$ and tumor necrosis factor alpha (TNF- $\alpha$ ) was then carried out according to the manufacturer's protocol (BD Biosciences) in combination with counterstaining using fluorochrome-conjugated anti-CD3, anti-CD4, and antiCD8 antibodies. A minimum of 150,000 CD3+ cell events were analyzed by multi-color flow cytometry. For proliferation assays, spleen cells were first labeled with CFSE $(0.5 \mu \mathrm{M})$ and then $2 \times 10^{5}$ cells/well co-cultured with either control DC or DC/AdV-RR5 at a ratio of 1:20 (DC/ spleen cells ratio) in the presence of low dose IL-2 (10 U/ 


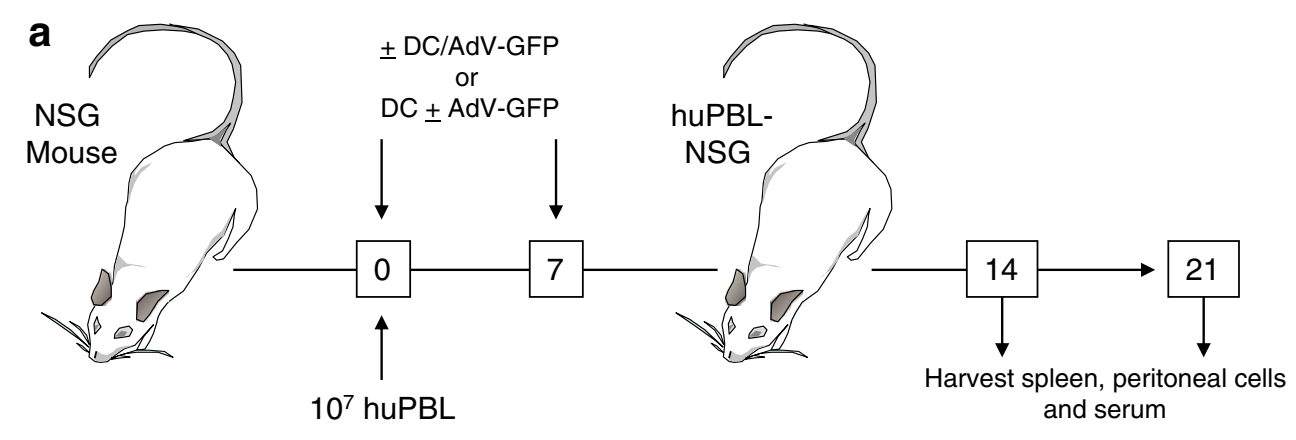

b huPBL

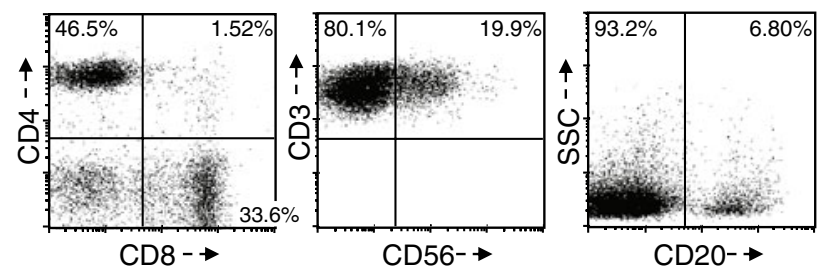

C Dendritic Cells (DC/AdV-GFP)
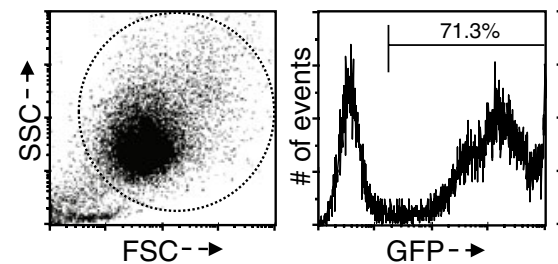

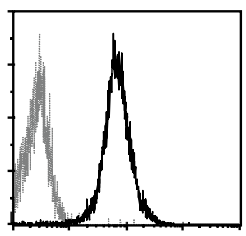

$\mathrm{CD} 11 \mathrm{c}-\rightarrow$

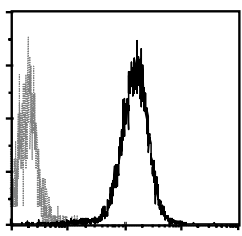

HLA-DR $\rightarrow$

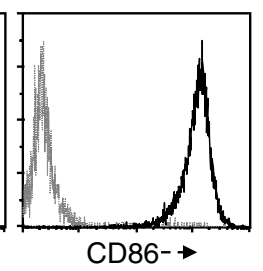

Fig. 1 Reconstitution of huPBL-NSG mice with both PBL and donormatched dendritic cells. a Six- to 8-week-old NSG mice were implanted with $10^{7}$ purified human T-and B-cells $(P B L)$ by i.p. injection. In order to restore antigen-presenting activity, animals were also administered donor-matched monocyte-derived DC alone or in combination with adenoviral antigens (as either DC/AdV-GFP or control DC + free AdV-GFP particles) by i.p. injection. In some experiments, animals received a single administration of $5 \times 10^{5} \mathrm{DC}$ and in other experiments they received a second dose of DC 7 days later. Spleens, peritoneal cells, and serum were recovered on day 14 or 21 for assessment of antigen-specific immune responses. b Purified human PBL used for implantation into NSG mouse were composed of

$\mathrm{ml})$ and IL-7 (1 ng/ml). Cells were harvested 5 days later, counterstained with fluorescent-conjugated $\mathrm{mAb}$ to indicated markers and analyzed by flow cytometry. Antigenspecific cytokine secretion was assessed by collecting supernatants from the same wells used for proliferation assays and the levels of human IFN- $\gamma$ and TNF- $\alpha$ measured by SearchLight multiplex assay (Thermo Scientific, Rockford, IL, USA).

In vivo cytokine production

Serum samples were collected from animals at the time of spleen recovery and assayed for the presence of subsets of T-cells (CD3+/CD4+, CD3+/CD8+, and CD3+/CD56+) and B-cells. c Monocyte-derived DC generated from the same donor express high levels of antigen and maturation markers. DC were transduced with AdV-GFP at an MOI of 4,000 viral particles/cell and were stained with antibodies against DC phenotype/maturation markers including CD11c, HLA-DR, and CD86 $48 \mathrm{~h}$ later. Flow cytometry demonstrated a uniform population of DC by forward scatter $(F S C)$ and side scatter (SSC). The percentage of GFP-positive cells was used to measure the percentage of DC expressing AdV antigens after transduction. The fluorescent profile of unstained DC is presented in gray and fluorescent staining for specific markers are depicted by black histogram curves. Representative experiment, $n=5$

human IFN- $\gamma$ and TNF- $\alpha$ to determine whether reconstitution with control DC versus DC/AdV-GFP resulted in detectable differences in systemic cytokine levels, reflecting the in vivo activation of antigenspecific T-cells.

\section{Statistical analysis}

Comparisons between groups were performed using Student's $t$ test, with $p<0.05$ accepted as a significant difference between groups. All experiments were performed with replicates and results from at least three separate experiments were pooled for statistical analysis. 


\section{Results}

Reconstitution of huPBL-NSG mice with both PBL and donor-matched dendritic cells

To adapt huPBL-NSG mice as a platform for assessing human antigen-specific T-cell activation, animals were implanted with an i.p. injection of $10^{7}$ human PBL and then supplemented with $0.5-1.0 \times 10^{6} \mathrm{DC}$ that had been generated from the same donor (donor-matched) as detailed in Fig. 1. Donor PBL were composed primarily of CD3+ Tcells with a normal complement of CD4 and CD8 subsets, a population of $\mathrm{CD} 3+/ \mathrm{CD} 56+$ cells, and $\mathrm{CD} 20+\mathrm{B}$-cells (Fig. 1b). Culture of adherent PBMC from the same donor with GM-CSF and IL-4, followed by immunomagnetic depletion of contaminating lymphocytes, resulted in a relatively pure and homogenous population of DC (Fig. 1c). DC prepared in this manner were highly susceptible to transduction by $\mathrm{AdV}$ and expressed high levels of the characteristic CD11c marker, MHC molecules (HLA-DR), and costimulatory molecules (such as CD86) required for efficient $\mathrm{T}$-cell activation.

Immune reconstitution of NSG mice with these two populations was associated with a marked migration to, and expansion of, human cells populating the spleen over the first 2-3 weeks. Splenic repopulation was associated with a concomitant decrease in the number of intraperitoneal cells (Fig. 2a). By the end of 3 weeks, the average yield of human cells from the spleen was $19.4 \pm 6.7 \times 10^{6}$ CD $45+$ cells and the number of residual CD45+ cells in the peritoneum had decreased to $1.9 \pm 0.6 \times 10^{6}$. After 14 days, $78.1 \pm 3.0 \% \quad(n=3)$ of recovered spleen cells expressed human CD45 and the percentage of human cells remained relatively constant through 21 days (Fig. 2b).

Recovered spleen cells contained a distribution of lymphocyte subsets similar to that of normal human blood including $\mathrm{CD} 3+/ \mathrm{CD} 4+, \mathrm{CD} 3+/ \mathrm{CD} 8+$, and $\mathrm{CD} 3+/ \mathrm{CD} 56+\mathrm{T}-$ cells along with a small population of B-cells $(\mathrm{CD} 20+)$ (Fig. 3a). The CD4/CD8 ratio averaged 1.63:1 $(n=15)$ and there was a direct relationship between the frequency of CD3+/CD56+ T-cells in the PBL used for implantation and those covered from the spleen $2-3$ weeks later $\left(r^{2}=0.85\right.$, $p<0.01)$. There was little evidence for T-cell activation as measured by the expression of either CD25 or CD69 (data not shown). The i.p. administration of mature monocytederived DC into huPBL-NSG mice also resulted in their migration and repopulation of the spleen by the end of 2 weeks. Similar to the mature phenotype of these cells at the time of administration, the majority of recovered CD11c+ splenic DC expressed high levels of HLA-DR and CD86 (>70\%) (Fig. 3b). huPBL-NSG mice reconstituted in this manner exhibited no physical signs of graft versus host disease.

\section{Activation of antigen-specific T-cells in vivo}

The capacity for human DC derived from the same donor to interact with and stimulate antigen-specific T-cells in vivo in huPBL-NSG animals was evaluated under two different conditions. In the first approach, animals were administered DC in combination with free AdV-GFP particles. In the second approach, animals were administered DC that had been loaded $48 \mathrm{~h}$ earlier with AdV capsid antigens in vitro. In both settings, the stimulation of memory T-cell responses to AdV capsid antigens was assessed by a standard 5-h ICCS assay in which nonspecific T-cell activation was assessed by challenging recovered cells with control DC (containing no antigen) while AdV-specific T-cell immunization was assessed by challenging with DC that had been loaded with the AdV/RR5 vector. Figure 4 demonstrates the results from a typical experiment. The baseline frequency of AdV-

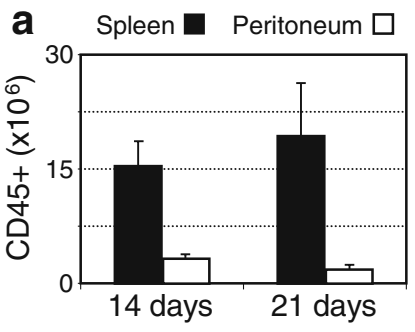

Fig. 2 Engraftment of NSG mice with human PBL. Six- to 8-weekold NSG mice were implanted with $10^{7}$ purified human T-and B-cells $(P B L)$ by i.p. injection. Spleen and peritoneal cells were recovered after 14 or 21 days, counted, and stained with anti-human CD45 mAb for analysis by flow cytometry. a The increase in human CD45+ leukocytes within the spleen from 14 to 21 days was associated with a

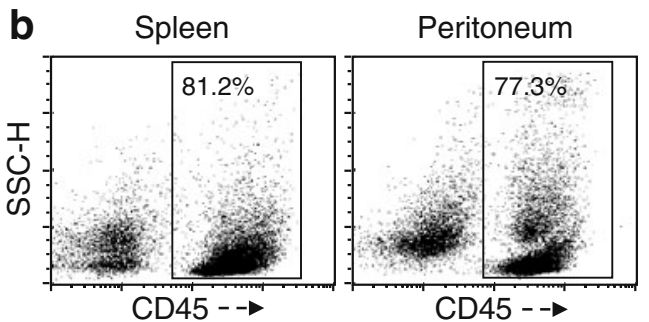

concomitant decrease in peritoneal cell number, suggesting a combined redistribution and expansion of human cells following i.p. implantation. b Representative dot-plots demonstrating the percentage of recovered cells from the spleen and peritoneum that expressed human CD45 at day 14. Average percentage of human CD45+ cells in the spleen at day 14 was $78.1 \pm 3.0 \%, n=3$ 
a Lymphocyte Subsets
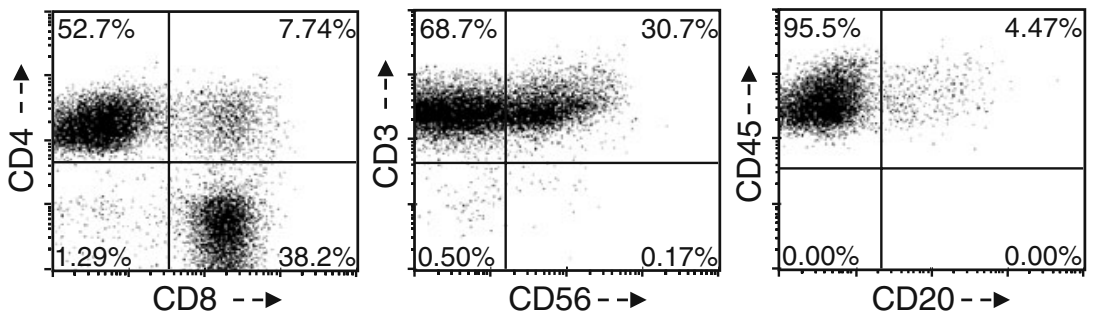

\section{b Dendritic Cells (CD11c+)}
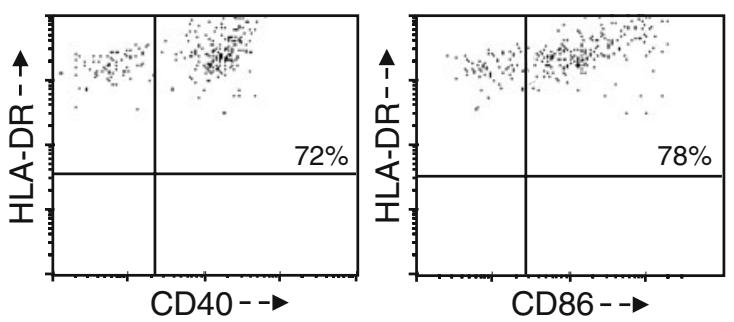

Fig. 3 Lymphocyte and DC phenotypes reconstituting the spleens of huPBL-NSG mice. a Six- to 8-week-old NSG mice were implanted with $10^{7}$ purified human PBL at day 0 in combination with $5 \times 10^{5}$ donor-matched monocyte-derived DC by i.p injection at day 0 and day 7. Spleens were recovered 14 days later and cells expressing human CD45 analyzed by flow cytometry. CD3+ T-cells exhibited a normal distribution of $\mathrm{CD} 4+$ and $\mathrm{CD} 8+$ subsets (left); a small population of B-cells was identified by their expression of CD20 (right); and NK-T-like cells were identified by their expression of

specific T-cells capable of producing IFN- $\gamma$ and TNF- $\alpha$ before implantation was measured at $0.10 \%$ of $\mathrm{CD} 4+\mathrm{T}-$ cells (all reported frequencies adjusted for the response to control DC) and $0.03 \%$ of CD8+ T-cells (Fig. 4a). When
CD56 alone or in combination with CD3, respectively (center). Representative experiment, $n=15$. b Six- to 8 -week-old NSG mice were implanted with $10^{7}$ purified human PBL at day 0 and $5 \times 10^{5}$ donor-matched monocyte-derived DC by i.p injection at day 7 . Spleens were recovered 14 days later and DC were identified by gating on the population of cells expressing CD11c and analyzing the $\mathrm{CD} 11 \mathrm{c}+$ subset for their expression of both HLA-DR and CD40 (left, gated for CD45+/CD11c+ cells) and both HLA-DR and CD86 (right, gated for $\mathrm{CD} 45+/ \mathrm{CD} 11 \mathrm{c}+$ cells). Representative experiment, $n=5$

PBL were administered to NSG mice in the absence of $\mathrm{DC}$, the frequency of AdV-specific CD4+ T-cells recovered from day 21 spleen cells was always less than $0.02 \%$ (data not shown). However, huPBL-NSG mice that were a

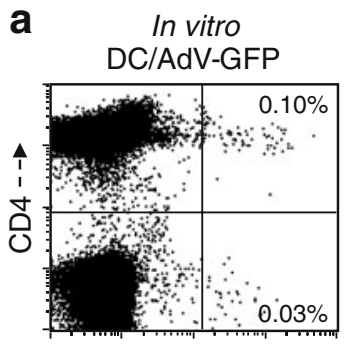

IFN- $\gamma /$ TNF- $\alpha \rightarrow$

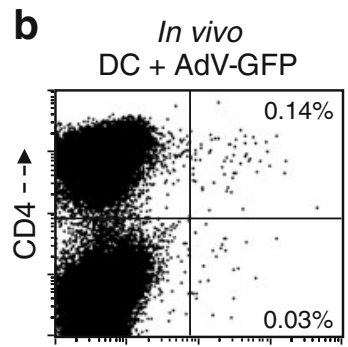

IFN- $\gamma /$ TNF- $\alpha-\rightarrow$

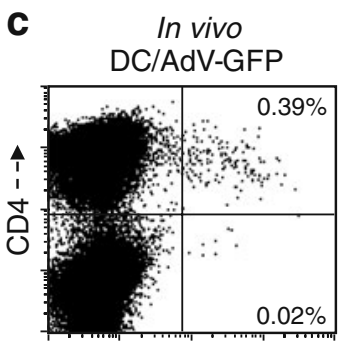

IFN- $\gamma /$ TNF- $\alpha \rightarrow$
Fig. 4 Donor-matched DC loaded with AdV antigens stimulate antigen-specific T-cell responses in vitro and in vivo in huPBL-NSG mice. Adherent PBMC were cultured with GM-CSF and IL-4 for 5 days, transduced with 4,000 MOI AdV-GFP (DC/AdV-GFP), with 4,000 MOI AdV-RR5 (DC/AdV-RR5), or cultured in transduction medium alone (control DC), and then matured with MegaCD40L and IFN- $\gamma$. a The capacity to stimulate antigen-specific T-cells in vitro was assessed by co-culturing DC/AdV-GFP with purified donormatched T-cells $(\mathrm{DC} / \mathrm{T}$ ratio $=1: 20)$ for 7 days followed by challenge with either control DC or DC/AdV-RR5 to detect AdV-specific T-cell frequency in a standard 5-h ICCS assay. Frequencies of antigenspecific CD4+ and CD8+ T-cells expressing IFN- $\gamma$ and/or TNF- $\alpha$ are noted, respectively (all frequencies are adjusted for the background response to control DC in the absence of antigen). Representative experiment, $n=3$. b, c The capacity for DC to stimulate antigenspecific T-cells in vivo in huPBL-NSG mice was similarly assessed by immunizing animals with either b $5 \times 10^{5} \mathrm{DC} / \mathrm{AdV}-\mathrm{GFP}$ (on day 7) or with c $5 \times 10^{5}$ control DC (on day 7) in combination with $2 \times 10^{9}$ free AdV-GFP particles. Spleen cells were harvested 14 days later and challenged in vitro with either control DC or DC/AdV-RR5 to detect AdV-specific T-cell frequency in a standard 5-h ICCS assay. Frequencies of antigen-specific CD4+ and CD8+ T-cells expressing IFN- $\gamma$ and/or TNF- $\alpha$ are noted, respectively. Representative experiment, $n=4$ 
immunized in vivo with the combination of DC and free AdV particles exhibited an AdV-specific CD4+ T-cell frequency of $0.14 \%$ (Fig. $4 b$ ). Furthermore, when huPBLNSG mice were immunized in vivo with DC/AdV-GFP, the frequency of AdV-specific CD4+ T-cells increased markedly to $0.39 \%$ (Fig. 4c). In replicate experiments, the $\mathrm{AdV}$-specific CD4+ T-cell responses in animals that were immunized with DC/AdV-GFP were always greater than the responses in animals that received control DC in combination with free AdV-GFP. When administering free AdV-GFP, the response to $10^{10}$ viral particles (shown in Fig. 4b) was generally greater than the response to $10^{9}$ viral particles (data not shown).

Antigen-specific cytokine production induced by DC-based immunization

Sera from immunized huPBL-NSG mice were tested for the presence of human IFN- $\gamma$ and TNF- $\alpha$ (Fig. 5). Compared to serum from control animals that were immunized with DC alone (no AdV exposure), serum from animals immunized with DC/AdV-GFP demonstrated consistently increased levels of both cytokines consistent with the responses measured in the ICCS assay.

Antigen-specific T-cells generated by immunization of huPBL-NSG mice respond to a secondary in vitro challenge

To further evaluate the function of antigen-specific T-cells that were activated in vivo, spleen cells were recovered from huPBL-NSG mice that were immunized with DC/AdV-GFP. The cells were labeled with CFSE and rechallenged in vitro for 5 days with either control DC (containing no antigens) or with DC/AdV-RR5 (Fig. 6). Although some background proliferation was observed in response to control DC, there was a clear antigen-specific increase in the percentage of proliferating $\mathrm{CD} 4+$ and CD4- T-cells in response to challenge with DC/AdV-RR5
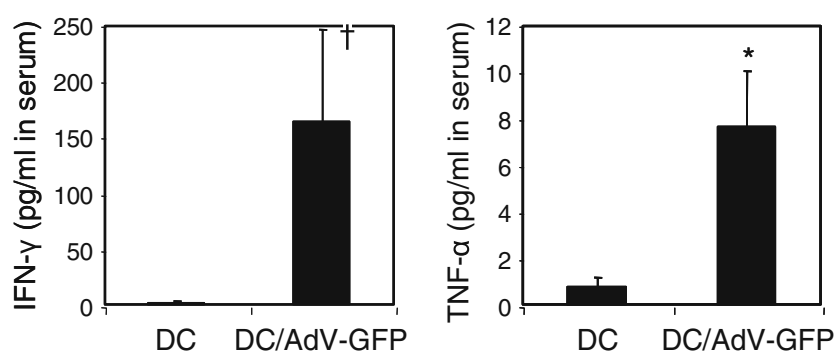

Fig. 5 In vivo cytokine response to DC/AdV-GFP. Sera from huPBLNSG mice were collected 14 days after immunization with either control DC or DC/AdV-GFP and assayed for human IFN- $\gamma$ and TNF$\alpha$ by SearchLight multiplex assay. Representative experiment, $n=3$. $\dagger p<0.10 ;{ }^{*} p \leq 0.05$

a In vitro stimulation of T-cells from huPBL-NSG mice previously immunized in vivo with $D C / A d V-G F P$

Control DC

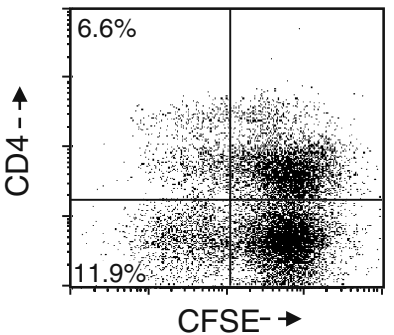

b Phenotype of proliferating T-cells (DC/AdV-RR5 group)

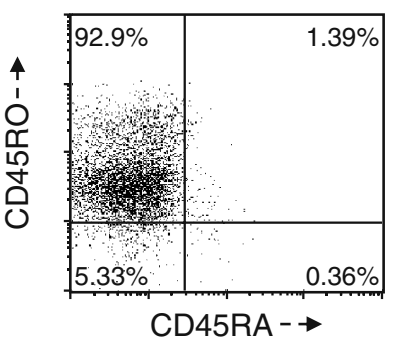

C In vitro cytokine production
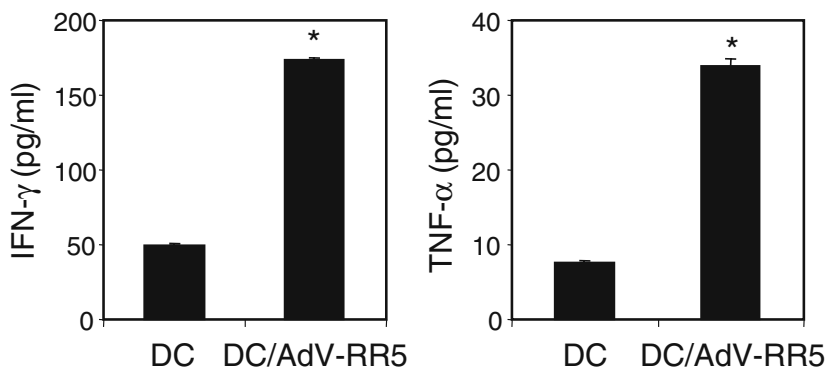

Fig. 6 T-cells recovered from immunized huPBL-NSG mice respond to antigen-specific stimulation in vitro. Spleen cells recovered from huPBL-NSG mice that were previously immunized with DC/AdVGFP in vivo were labeled with CFSE and co-cultured with either control DC or DC/AdV-RR5 (DC/T ratio=1:20) for 5 days in vitro. a Cells were stained with anti-CD3 and anti-CD4 and the CD3+ T-cells analyzed for adenoviral-specific proliferative responses by flow cytometry. Representative experiment, $n=5$. b The sub-population of cells proliferating in response to stimulation with DC/AdV-RR5 (CFSE-dim) were further analyzed for their expression of effector/ memory and regulatory T-cell markers. c Supernatants from the same wells were harvested and assessed for the presence of human IFN- $\gamma$ and TNF- $\alpha$ by SearchLight multiplex assay. Representative experiment, $n=3$. ${ }^{*} p<0.01$

(Fig. 6a). Further phenotyping of the proliferating cells revealed that they expressed CD45RO, a memory cell marker, and that a significant percentage expressed CD25, an activation marker, in the absence of GITR, suggesting that they were not suppressor cells (Fig. 6b). Consistent with the expansion of effector/memory T-cells, supernatant collected from these co-cultures demonstrated antigenspecific induction of IFN- $\gamma$ and TNF- $\alpha$ (Fig. $6 \mathrm{c}$ ). 


\section{Discussion}

Fundamental differences between the mouse and human immune systems, differences in genetic diversity, as well as inherent differences in the susceptibility and exposure to human disease, often limit the translational potential of mouse models for developing effective treatments for human disease. A variety of "humanized" animal models have been developed to address these issues by either expressing human genes/traits in animals (Pascolo et al. 1997; Mangalam et al. 2008; Conforti et al. 2009) or by engrafting human immune cells into immunodeficient mice (Mosier et al. 1988; Ito et al. 2002; Ishikawa et al. 2005). However, one significant limitation has been the study of adaptive immunity. The huPBL-NSG model provides a unique opportunity to study the interaction of various immune challenges with human immune cells (Kumar et al. 2008; Strowig et al. 2009; King et al. 2009). We herein demonstrate that the addition of donor-matched monocyte-derived DC to the reconstitution of huPBL-NSG animals enabled us to rapidly assess and stimulate the donor's antigen-specific memory in vivo, in the intact animal, as well as ex vivo, after recovery of spleen cells from immunized animals.

Our results confirm that rapid reconstitution of the spleen and peripheral blood occurs following a single i.p. injection of human PBL. Within a few weeks, 15-25 million human cells can be recovered from the spleen with the $\mathrm{CD} 3+/ \mathrm{CD} 4+, \mathrm{CD} 3+/ \mathrm{CD} 8+, \mathrm{CD} 3+/ \mathrm{CD} 56+$, and $\mathrm{CD} 20+$ populations all well represented in appropriate proportions. Regardless of whether administered only once (at day 0 or 7) or twice (at both day 0 and 7), implanted DC were detected at low levels in the spleen and expressed the phenotypic markers of functional antigen-presenting cells. Importantly, reconstitution with DC did not lead to physical signs of GVHD or to changes in the level of acute activation markers (CD25 and CD69; data not shown). However, reconstitution with DC did enable the huPBLNSG animals to respond to antigen challenge. Adenovirus was chosen as the target antigen for this work due to its well-characterized immunogenicity in humans and the presence of highly sensitive assays for assessing AdVspecific T-cell responses (Roth et al. 2002). Similar to the response pattern observed in vivo when humans are immunized with AdV (Gahéry-Ségard et al. 1997), fully reconstituted huPBL-NSG animals rapidly expanded the population of $\mathrm{AdV}$-specific T-cells following exposure to $\mathrm{AdV}$. In the absence of an antigen challenge, essentially no detectable AdV-specific T-cells were detected in freshly harvested splenocytes. However, following active immunization with DC/AdV-GFP, there was a dramatic upregulation of AdV-specific T-cells that far exceeded the level detected when the original donor PBL were stimulated in vitro for a week with IL-2, IL-7, and DC/AdV-GFP. As might be expected when healthy patients are immunized with a vaccine, immunization of the intact animal was more effective than standard in vitro stimulation. While a cellbased vaccination (DC/AdV-GFP) elicited the greatest in vivo response, administration of free AdV-GFP vector also stimulated an AdV-specific T-cell response. In this case, the magnitude of the response appeared to increase with the quantity of antigen administered.

In addition to the in vivo expansion of antigen-specific T-cells, immunization of huPBL-NSG animals with DC/ AdV-GFP induced the systemic release of IFN- $\gamma$ and TNF$\alpha$ into the serum. The detection of these circulating cytokines was a robust indicator of the AdV-specific Tcell response and consistent with reports that IFN- $\gamma$ and TNF- $\alpha$ represent the predominant T-cell cytokines produced upon AdV challenge (Hutnick et al. 2010). It is noteworthy that resting cytokine levels for IFN- $\gamma$ and TNF$\alpha$ were low in unchallenged animals, arguing against the presence of spontaneous responses to endogenous mouse antigens.

One advantage of the huPBL-NSG mouse compared to its NOD/SCID precursors is the robust repopulation of the spleen with human cells. Harvesting spleens from these animals provides an abundant source of T-cells for in vitro analysis. As occurs when human T-cells are recovered from the blood of healthy subjects, the T-cells recovered from the spleens of immunized animals were able to undergo further expansion and testing when stimulated with $\mathrm{AdV}$ for another 5-7 days in vitro. Labeling recovered spleen cells with CFSE allowed us to track the proliferative response to a secondary in vitro challenge with DC/AdV-RR5 and to characterize the phenotype of responding cells. Interestingly, this was the one situation in which there was a significant background response when the cells were stimulated with control DC that had not been loaded with AdV. As the co-cultures contained up to $30 \%$ mouse cells recovered from the spleen, it seems likely that uptake and processing of these cells by the added DC lead to an in vitro anti-mouse response. However, despite this higher background proliferation, challenging in vitro with $\mathrm{DC} / \mathrm{AdV}$ RR5 still led to a significantly greater response than did challenging with control DC alone. T-cells proliferating in response to DC/AdV-RR5 were mostly CD45RO+ cells and approximately $25 \%$ of them expressed moderate levels of CD25 without expression of GITR, suggesting the presence of memory/effector T-cells, but not T-regulatory cells. This would be expected given our use of mature DC to immunize the animals in vivo (Roth et al. 2002; Harui et al. 2004). As detected in serum collected from immunized animals, IFN- $\gamma$ and TNF- $\alpha$ were secreted into the culture supernatant during the in vitro challenge.

In summary, these findings support our hypothesis that reconstitution of NSG mice with a combination of human 
PBL and mature donor-matched human DC results in a huPBL-NSG model in which adaptive T-cell responses can be stimulated and measured in the intact animal. There are several potential advantages of this model over others that have been reported in the literature. Gorantla et al. (2005) reported that NOD/SCID animals can be treated with asialo-GM1 and anti-CD122 antibodies to aid human engraftment and then vaccinated with antigen-loaded DC. While providing an important proof of concept, the use of NOD/SCID animals for this purpose has been replaced by newer animal strains due to the large number of cells required and the very limited engraftment that is obtained (Shultz et al. 2000; Shultz et al. 2003). Conventional engraftment of NSG mice with CD34+ stem cells is limited by the failure of normal T-cell development and the lack of pre-existing immune memory (Ishikawa et al. 2005). Yu et al. (2008) overcame these limitations by transplanting NOD/SCID $\beta 2$ microglobulin ${ }^{-} /$mice with peripheral blood CD34+ stem cells $\left(3 \times 10^{6} /\right.$ animal $)$ to promote DC and B-cell repopulation followed by supplementation 4-8 weeks later with donor-matched PBL as the source of T-cell responders. While requiring a leukopheresis to obtain sufficient CD34 donor cells, whole-body irradiation in combination with a 10-day course of human FLT3 ligand to promote engraftment, and then a protracted period for reconstitution, the resulting humanized animals responded well to both live influenza virus and inactivated influenza vaccine. As in our model, they observed a significant in vivo expansion of memory/effector T-cells with defined antigen specificity and cytokine production. Also similar to our work, they demonstrated that DC were crucial for the generation of an antigen-specific response. While based on similar principals, our model allows reconstitution from a single peripheral blood draw, and animals are ready for immunologic testing in as little as 2 weeks without further manipulation. In addition, these animals are stable for at least 6 weeks after implantation of human PBL. The large number of human cells that can be recovered from the spleen facilitates the extensive in vitro analysis that is often required when mapping out T-cell responses. Altogether, this model provides a highly adaptable platform to study the interaction of antigen presentation and antigen-specific T-cell responses in vivo. Immunologic responses to a variety of viruses can likely be studied using these approaches, new vaccines tested without the need to administer them to human subjects, and a variety of drugs and immune modulators evaluated for their impact on various stages of antigen presentation and T-cell function.

Conflict of interest statement The authors do not have financial disclosure, personal relationship, or known conflict of interest related to this work.
Open Access This article is distributed under the terms of the Creative Commons Attribution Noncommercial License which permits any noncommercial use, distribution, and reproduction in any medium, provided the original author(s) and source are credited.

\section{References}

Conforti A, Peruzzi D, Giannetti P, Biondo A, Ciliberto G, La Monica N, Aurisicchio L (2009) A novel mouse model for evaluation and prediction of HLA-A2-restricted CEA cancer vaccine responses. J Immunother 32(7):744-54

Gahéry-Ségard H, Molinier-Frenkel V, Le Boulaire C, Saulnier P, Opolon P, Lengagne R, Gautier E, Le Cesne A, Zitvogel L, Venet A, Schatz C, Courtney M, Le Chevalier T, Tursz T, Guillet JG, Farace F (1997) Phase I trial of recombinant adenovirus gene transfer in lung cancer. Longitudinal study of the immune responses to transgene and viral products. J Clin Invest 100 (9):2218-26

Gorantla S, Santos K, Meyer V, Dewhurst S, Bowers WJ, Federoff HJ, Gendelman HE, Poluektova L (2005) Human dendritic cells transduced with herpes simplex virus amplicons encoding human immunodeficiency virus type 1 (HIV-1) gp120 elicit adaptive immune responses from human cells engrafted into NOD/SCID mice and confer partial protection against HIV-1 challenge. J Virol 79(4):2124-32

Greiner DL, Hesselton RA, Shultz LD (1998) SCID mouse models of human stem cell engraftment. Stem Cells 16(3):166-77

Harui A, Roth MD, Kiertscher SM, Mitani K, Basak SK (2004) Vaccination with helper-dependent adenovirus enhances the generation of transgene-specific CTL. Gene Ther 11 (22): $1617-26$

Harui A, Roth MD, Vira D, Sanghvi M, Mizuguchi H, Basak SK (2006a) Adenoviral-encoded antigens are presented efficiently by a subset of dendritic cells expressing high levels of alpha(v)beta3 integrins. J Leukoc Biol 79(6):1271-8

Harui A, Roth MD, Sanghvi M, Vira D, Mizuguchi H, Basak SK (2006b) Centrifugation enhances integrin-mediated transduction of dendritic cells by conventional and RGD-modified adenoviral vectors. J Immunol Methods 312(1-2):94-104

Hesselton RM, Greiner DL, Mordes JP, Rajan TV, Sullivan JL, Shultz LD (1995) High levels of human peripheral blood mononuclear cell engraftment and enhanced susceptibility to human immunodeficiency virus type 1 infection in NOD/LtSz-scid/scid mice. J Infect Dis 172(4):974-82

Hutnick NA, Carnathan D, Demers K, Makedonas G, Ertl HC, Betts MR (2010) Adenovirus-specific human T-cells are pervasive, polyfunctional, and cross-reactive. Vaccine 28(8):1932-41

Ishikawa $\mathrm{F}$, Yasukawa M, Lyons $\mathrm{B}$, Yoshida S, Miyamoto $\mathrm{T}$, Yoshimoto G, Watanabe T, Akashi K, Shultz LD, Harada M (2005) Development of functional human blood and immune systems in NOD/SCID/IL2 receptor gamma chain (null) mice. Blood 106(5):1565-73

Ito M, Hiramatsu H, Kobayashi K, Suzue K, Kawahata M, Hioki K, Ueyama Y, Koyanagi Y, Sugamura K, Tsuji K, Heike T, Nakahata T (2002) NOD/SCID/gamma(c)(null) mouse: an excellent recipient mouse model for engraftment of human cells. Blood 100(9):3175-82

Ju X, Clark G, Hart DN (2010) Review of human DC subtypes. Meth Mol Biol 595:3-20

Kiertscher SM, Roth MD (1996) Human CD14+ leukocytes acquire the phenotype and function of antigen-presenting dendritic cells when cultured in GM-CSF and IL-4. J Leukoc Biol 59(2):208-18 
King MA, Covassin L, Brehm MA, Racki W, Pearson T, Leif J, Laning J, Fodor W, Foreman O, Burzenski L, Chase TH, Gott B, Rossini AA, Bortell R, Shultz LD, Greiner DL (2009) Human peripheral blood leucocyte non-obese diabetic-severe combined immunodeficiency interleukin-2 receptor gamma chain gene mouse model of xenogeneic graft-versus-host-like disease and the role of host major histocompatibility complex. Clin Exp Immunol 157(1):104-18

Kumar P, Ban HS, Kim SS, Wu H, Pearson T, Greiner DL, Laouar A, Yao J, Haridas V, Habiro K, Yang YG, Jeong JH, Lee KY, Kim YH, Kim SW, Peipp M, Fey GH, Manjunath N, Shultz LD, Lee SK, Shankar P (2008) T cell-specific siRNA delivery suppresses HIV-1 infection in humanized mice. Cell 134(4):577-86

Lapidot T, Pflumio F, Doedens M, Murdoch B, Williams DE, Dick JE (1992) Cytokine stimulation of multilineage hematopoiesis from immature human cells engrafted in SCID mice. Science 255 (5048):1137-41

Mangalam AK, Rajagopalan G, Taneja V, David CS (2008) HLA class II transgenic mice mimic human inflammatory diseases. Adv Immunol 97:65-147

May KF Jr, Roychowdhury S, Bhatt D, Kocak E, Bai XF, Liu JQ, Ferketich AK, Martin EW Jr, Caligiuri MA, Zheng P, Liu Y (2005) Anti-human CTLA-4 monoclonal antibody promotes Tcell expansion and immunity in a hu-PBL-SCID model: a new method for preclinical screening of costimulatory monoclonal antibodies. Blood 105(3):1114-20

Mestas J, Hughes CC (2004) Of mice and not men: differences between mouse and human immunology. J Immunol 172:2731-2738

Mittereder N, March KL, Trapnell BC (1996) Evaluation of the concentration and bioactivity of adenovirus vectors for gene therapy. J Virol 70(11):7498-509

Mizuguchi H, Koizumi N, Hosono T, Utoguchi N, Watanabe Y, Kay MA, Hayakawa T (2001) A simplified system for constructing recombinant adenoviral vectors containing heterologous peptides in the HI loop of their fiber knob. Gene Ther 8(9):730-5

Mosier DE, Gulizia RJ, Baird SM, Wilson DB (1988) Transfer of a functional human immune system to mice with severe combined immunodeficiency. Nature 335:256-259

Olive M, Eisenlohr LC, Flomenberg P (2001) Quantitative analysis of adenovirus-specific CD4+ T-cell responses from healthy adults. Viral Immunol 14(4):403-13

Pascolo S, Bervas N, Ure JM, Smith AG, Lemonnier FA, Pérarnau B (1997) HLA-A2.1-restricted education and cytolytic activity of
CD8(+) $\mathrm{T}$ lymphocytes from beta2 microglobulin (beta2 $\mathrm{m}$ ) HLA-A2.1 monochain transgenic $\mathrm{H}-2 \mathrm{Db}$ beta2m double knockout mice. J Exp Med 185(12):2043-51

Pearson T, Greiner DL, Shultz LD (2008) Humanized SCID mouse models for biomedical research. Curr Top Microbiol Immunol $324: 25-51$

Roth MD, Cheng Q, Harui A, Basak SK, Mitani K, Low TA, Kiertscher SM (2002) Helper-dependent adenoviral vectors efficiently express transgenes in human dendritic cells but still stimulate antiviral immune responses. J Immunol 169 (8):4651-6

Shultz LD, Lang PA, Christianson SW, Gott B, Lyons B, Umeda $\mathrm{S}$, Leiter E, Hesselton R, Wagar EJ, Leif JH, Kollet $\mathrm{O}$, Lapidot T, Greiner DL (2000) NOD/LtSz-Rag1null mice: an immunodeficient and radioresistant model for engraftment of human hematolymphoid cells, HIV infection, and adoptive transfer of NOD mouse diabetogenic T-cells. J Immunol 164:2496-2507

Shultz LD, Banuelos S, Lyons B, Samuels R, Burzenski L, Gott B, Lang P, Leif J, Appel M, Rossini A, Greiner DL (2003) NOD/LtSz-Rag1nullPfpnull mice: a new model system to increase levels of human peripheral leukocyte and hematopoietic stem cell engraftment. Transplantation 76:1036-1042

Shultz LD, Lyons BL, Burzenski LM, Gott B, Chen X, Chaleff S, Kotb M, Gillies SD, King M, Mangada J, Greiner DL, Handgretinger R (2005) Human lymphoid and myeloid cell development in NOD/ LtSz-scid IL2R gamma null mice engrafted with mobilized human hemopoietic stem cells. J Immunol 174(10):6477-89

Shultz LD, Ishikawa F, Greiner DL (2007) Humanized mice in translational biomedical research. Nat Rev Immunol 7(2):118-30

Strowig T, Gurer C, Ploss A, Liu YF, Arrey F, Sashihara J, Koo G, Rice CM, Young JW, Chadburn A, Cohen JI, Münz C (2009) Priming of protective T-cell responses against virus-induced tumors in mice with human immune system components. J Exp Med 206(6):1423-34

Yu CI, Gallegos M, Marches F, Zurawski G, Ramilo O, García-Sastre A, Banchereau J, Palucka AK (2008) Broad influenza-specific CD8+ T-cell responses in humanized mice vaccinated with influenza virus vaccines. Blood 112(9):3671-8

Zaiss AK, Machado HB, Herschman HR (2009) The influence of innate and pre-existing immunity on adenovirus therapy. J Cell Biochem 108(4):778-90 